\title{
UNA APROXIMACIÓN BIOÉTICA Y DE DERECHOS HUMANOS A LA INTERSEXUALIDAD EN COSTA RICA
}

\section{AN APPROACH TO INTERSEXUALITY IN COSTA RICA FROM BIOETHICS AND HUMAN RIGHTS}

\author{
José Joaquín Alvarado Acuña \\ Investigador independiente \\ jalvaradoa@hotmail.com
}

Publicado 2016

\section{Resumen \\ El problema medular, abordado en este artículo, surge de la preocupación por la inexistencia de información y de protocolos de atención para las personas intersexuales, especialmente para menores de edad o recién nacidos, por su invisibilización social, los estigmas que pesan sobre ellos y la medicalización o patologización de los individuos intersexuales, producién- dose de ese modo su cosificación y deshumanización.}

Palabras clave: intersexualidad, bioética, bioderecho, derechos humanos.

\section{Summary}

The main problem addressed in this article starts in the concern about the lack of information and care protocols for intersex people, especially minors or newborns, their social invisibility, the stigma hanging over them and the medicalization and pathologization of intersex individuals, thereby producing its objectification and dehumanization.

Keywords: intersexuality, bioethics, bio law, human rights.

\section{Introducción}

En reiteradas oportunidades he cuestionado el origen de diversas situaciones que sufren algunos individuos con ciertas características, así como las posibles razones para que, desde la sociedad, la cultura, la política o la 
religión, entre otras, se les discrimine aunque en nada difieran, en tanto seres humanos, de quienes se dedican a prácticas discriminatorias.

He cuestionado las estructuras del poder, su posicionamiento, influencia, manifestación, y discurso, las falacias ocultas para fundamentarse, el lenguaje utilizado para perpetuar el trato cruel, degradante y discriminatorio, así como la masificación de las reacciones populares ante llamados a oposiciones civiles y "guerras santas" que nos libren de lo que se disfraza de "amenaza" a la humanidad. Lo anterior al cuestionar el sentido del concepto de "ser humano", y se encuentra razón alguna para establecer tales diferencias desde el iusnaturalismo, el iuspositivismo, la ética o la moral.

Me explico brevemente: existe tanta diversidad en "Lo humano", tantos matices que nutren y surgen de esa condición, que debe contenerse con presión política, social, cultural, económica y religiosa. Aún más, surge el cuestionamiento sobre el porqué a toda esa diversidad de "Lo humano" se le permite manifestarse solamente en dos únicas formas: masculina o femenina.

Y es que entre estas manifestaciones de "Lo humano" encontramos a las personas intersexuales, a quienes, a lo largo de la historia, se les ha denominado de distintas maneras: hermafroditas, (monstruos en inglés), indefinidos, intersexuales; y a su duplicidad genital se le ha denominado recientemente "desórdenes del desarrollo sexual" (DSD por sus siglas en inglés).

Una mejor definición y el uso adecuado de este vocabulario permitirán romper lingüísticamente con lo que el discurso de poder ha establecido como parámetros de normalidad y anormalidad. En palabras de Berger y Luckmann (2001) “(...) el lenguaje marca las coordenadas de mi vida en la sociedad y llena esa vida de objetos significativos" (p. 39). De ahí que, desde este momento, hago la aclaración de que las referencias y terminología médicas que se incluyan en este trabajo son absolutamente ilustrativas del uso inadecuado de los vocablos, y expuestas con miras a evidenciar aspectos propios de un discurso que perpetúa la vulneración de estas personas.

Poco se ha dicho sobre la intersexualidad en nuestro país, pero en Latinoamérica, actualmente, este tema ha tomado los titulares periodísticos, en virtud de una tendencia mundial a cambiar el paradigma existente, por el cual es obligatorio que la asignación del sexo legal deba hacerse a partir de la genitalidad. 
El cambio radica en el enfoque del tema: pasar de lo que conceptualmente se ha estandarizado como "sexo legal", basado en la exterioridad, en la apariencia física de los órganos sexuales del individuo, su genitalidad, a una fijación autónoma -por parte del individuo-del sexo genotípico, aquel con el que esa persona, y solamente esa persona, se identifica, su íntima identidad de género.

Debe comprenderse que la intersexualidad ha sido relacionada con la apariencia externa del aparato genitourinario, otrora conceptualizada como hermafroditismo, pues hace referencia al poema de Ovidio, Metamorfosis, en el cual se narra el tránsito del hijo varón de Hermes y Afrodita a un ser que, luego de unirse sexualmente con una náyade, conjugó en sí mismo ambos sexos, y cuyo nombre finalmente fue Hermafrodito.

Los médicos han utilizado el término o connotación de "hermafrodita", para referirse a aquellas personas que, por haber nacido con apariencia de duplicidad en sus genitales -externos, internos o ambos-, requieren un procedimiento "correctivo de su sexo físico". Este discurso médico ha sido utilizado, históricamente, para convencer a los progenitores sobre la necesidad de intervención de este "fenómeno" en edades tempranas y, luego, para discriminar, ocultar e incluso convertir a tales personas en seres anormales, de circo, y en objetos de experimentación médica (clínica, quirúrgica y psiquiátrica).

\section{Aspectos médicos sobre la intersexualidad}

Los estudios epidemiológicos, así como la investigación médica, han podido determinar coincidencias entre la (HSC) y la intersexualidad. Médicamente, la intersexualidad se define como "un grupo de afecciones donde hay una discrepancia entre los genitales internos y externos (los testículos y los ovarios)" (MedlinePlus, 2014, s. p.). Además, con miras a evitar algunas confusiones relacionadas con esta condición, y los estigmas que produce, se ha empezado a migrar hacia el concepto de "desórdenes del desarrollo sexual" ("DSD", por sus siglas en inglés).

Según el sitio web de la Biblioteca Nacional de Medicina de Estados Unidos, en conjunto con los Institutos Nacionales de Salud (NIH), conocido como MedlinePlus, la intersexualidad se puede dividir en cuatro categorías: "Intersexualidad 46, XX"; "Intersexualidad 46, XY"; "Intersexualidad gonadal 
verdadera"; e "Intersexualidad compleja o indeterminada" (MedlinePlus, 2014). La más común de todas es la hiperplasia suprarrenal congénita (HSC) (MedlinePlus, 2014).

Finalmente, de los trastornos de intersexualidad compleja o indeterminada del desarrollo sexual, se afirma que:

muchas configuraciones de cromosomas distintos a las combinaciones simples 46, XX o 46, XY pueden ocasionar trastornos del desarrollo sexual y abarcan, entre otros, 45, XO (solamente un cromosoma X), y 47 XXY, 47 XXX: ambos casos tienen un cromosoma sexual adicional, sea un $\mathrm{X}$ o un $\mathrm{Y}$. Estos trastornos no ocasionan una condición de intersexualidad donde haya discrepancia entre órganos genitales externos e internos. Sin embargo, puede haber problemas con los niveles de hormonas sexuales, el desarrollo sexual en general y alteración en los números de cromosomas sexuales (MedlinePlus, 2014).

"Epidemiológicamente", se ha logrado establecer una media aproximada de incidencia de esta condición en el mundo, de 1-2: 2000 individuos nacidos vivos (Wilchins, 2001).

Según un artículo publicado por Silvia Castillo Nieto, en la Revista Dominical del periódico, el 23 de noviembre de 1997:

Costa Rica ocupa el tercer puesto en el mundo en la incidencia de hiperplasia suprarrenal congénita (...). Las estadísticas demuestran que uno de cada 4.000 nacidos vivos en el país presenta, de forma menos o más severa, ambigüedad sexual al momento de nacer. El primer lugar le corresponde a una población de esquimales en Alaska y el segundo, a la isla La Reunión, una colonia francesa. En Costa Rica, existen zonas donde la incidencia es más marcada, como Grecia, Sarchí, Orotina y las faldas del volcán Poás. Por su parte, Limón es la provincia con menos casos registrados de hiperplasia suprarrenal (Castillo Nieto, 1997, s. p.).

De lo anterior, se puede concluir que la población de Costa Rica no está exenta de padecer de hiperplasia suprarrenal congénita y, por ende, de que tengamos un porcentaje importante de población intersexual, lo que es extraño, pues no existen registros médicos ni estadísticos sobre la incidencia de nacimientos vivos intersexuales. 


\section{Aspectos bioéticos sobre las prácticas médicas de la intersexualidad Clínica}

La determinación inicial de un estado intersexual no se produce únicamente por medio de la observación del médico. Existe todo un abanico de "pruebas" que se realizan o, al menos, así se describe en la literatura que se revisó.

En un artículo publicado en la, de setiembre de 2013, denominado Desórdenes del Desarrollo Sexual y Cirugía Correctiva, se incluye una explicación sobre algunos de los procesos médicos para "lograr una corrección adecuada de reasignación de sexo", y se exalta "la tecnología existente y los diversos mecanismos con que se cuenta", como un plus para lograr "un adecuado y oportuno diagnóstico, con el objetivo de alcanzar lo antes posible la reasignación de sexo más adecuada" (Alemán Ramírez ., 2013).

$\mathrm{Al}$ respecto, indican que para definir estos estados de intersexualidad se debe considerar si la persona tiene determinado su sexo de acuerdo con los componentes cromosómico, gonadal, genital y social ya descritos. Es por esto que los métodos utilizados para determinar si una persona tiene genitales ambiguos están asociados a una serie de investigaciones entre las que se encuentran:

- De tipo genéticas: cariotipo.

- De tipo endocrino: pruebas de sangre, orina y ensayos de hormonas.

- De tipo radiológico: ultrasonidos (pélvicos), ecografía abdómino-pelviana.

- De exploración: exploración de genitales externos (tamaño del clítoris y pene, separación de los labios mayores, bolsas escrotales, situación del meato urinario, abocamiento vaginal, palpación de las gónadas).

- De tipo quirúrgicos: laparoscopía y biopsias gonadales. (Chandrasen, 2010).

Cuando se trata de la presencia de genitales ambiguos, es obligatoria la realización del cariotipo, el cual se lleva a cabo principalmente con la sangre periférica, a partir de los leucocitos, además de establecerse la existencia del gen SRY. Con respecto al análisis endocrino, este se basa, principalmente, en el estudio de esteroides suprarrenales y gonadales de la hormona adrenocorticotropa (ACTH) y el cortisol, para valorar la posibilidad de hiperplasia suprarrenal congénita, la cual es una de las principales causas de este trastorno y puede poner en riesgo la vida del neonato. Los exámenes de tipo 
radiológico son de gran ayuda para identificar el útero, ovarios y testículos abdominales o en el trayecto inguinal (Pelayo ., 2011).

Y esto es solo el inicio, pues luego sigue lo más interesante: los tratamientos.

\section{Tratamientos}

El mismo artículo cita cómo otras técnicas, usadas en personas intersexuales, ayudan a "corregir" el sexo físico inclinado hacia órganos externos masculinos o femeninos. Estas, según Alemán Ramírez y otros (2013) y Selvaggi y Bellringer (2011), son:

\section{Cirugía de feminización}

- Reducción del clítoris: Las técnicas más destacadas pretenden conservar aspectos sensoriales y cosméticos del clítoris. En casos severos de masculinización de los genitales (síndrome de Prader V) puede considerarse la posibilidad de reducir la cantidad de tejido eréctil.

- $\quad$ Separación de vagina y uretra: La duplicación vaginal al lado es mucho más frecuente y se realiza la sección del tabique en línea media. Pueden usarse colgajos cutáneos para la fijación vaginal.

- Vaginoplastia: El tipo de vaginoplastia depende del nivel de masculinización. Para altas anomalías del seno urogenital, el uso del alargado seno urogenital común como un colgajo vaginal anterior puede ser necesario, así como la sustitución con un injerto de piel o intestino tal vez sea necesario.

\section{Cirugía de masculinización}

- Orquidopexia: Una bolsa escrotal vacía implica uno o ambos testículos no descendidos. Con esta cirugía se intenta descender los testículos dentro del escroto.

- Faloplastia: Varias técnicas se han ideado para lograr una reconstrucción de los genitales masculinos, entre ellas microanastomosis libre de inervados radiales del antebrazo y las del recto abdominal miocutáneo. Sin embargo, se da mayor énfasis 
a nuevas técnicas de ingeniería de tejidos. Las principales complicaciones de estos procedimientos son la fístula, la erosión de la prótesis, y la sensación pobre.

De lo dicho hasta ahora se deducen dos cosas. Primero, que la HSC tiene altos niveles de prevalencia en el país, somos el tercer puesto en incidencia de esta enfermedad. Si la población nacional asciende a 4301712 personas $^{1}$ y la media de personas intersexuales es de 1-2: 2000 personas, cuando menos 4302 personas han nacido intersexuales en Costa Rica, o sea un $0.1 \%$ de la población. Segundo, que aunque porcentualmente parezca una cifra muy baja, la presencia de esta condición en la población no puede pasar desapercibida, no solo por los casos de HSC con virilización o feminización en neonatos, sino porque, siendo hereditaria, la incidencia de casos puede crecer exponencialmente desde los progenitores que porten la HSC sin saberlo, se casen y reproduzcan, y sin que haya indicadores favorables -en la relación riesgo beneficio-, para el tratamiento prenatal con corticoides, lo que remarca la importancia actual del tema.

No obstante, en la literatura nacional, curiosamente, apenas se ubica alguna estadística relativa a los casos de intersexualidad, en el Área de Estadística en Salud de la Caja Costarricense de Seguro Social, Dirección de Proyección de Servicios de Salud, y sobre su tratamiento. Según Carmen Di Mare Hering, jefe del Área de Bioética del CENDEISS de la Caja Costarricense de Seguro Social, no existe, institucionalmente, guía de tratamiento alguna.

Esto puede ser porque las decisiones en torno a este "trastorno físico de orden genético" se toman, por parte de los progenitores, a edades muy tempranas de los individuos que nacen con duplicidad genital y, por ello, no se cuantifican los individuos vivos con esta condición. Esto implica una suerte de invisibilización de estas poblaciones, a partir de un mal entendido "mecanismo de protección", en beneficio del menor y de su futura vida.

Por otra parte, quizá se deba a que la intervención del médico en la reasignación del sexo genital, producto de un discurso basado en una visión binaria del sexo, heteronormativo, hegemónico y normalizador, con autoridad suficiente para imponerse a los progenitores y a los pacientes, incida en

1 Según la última encuesta nacional del Instituto Nacional de Estadística y Censos de Costa Rica, al 2011 la población total era de 4301712 personas. Fuente: http://www.inec.go.cr/Web/Home/pagPrincipal.aspx\# 
la decisión de los primeros sobre su prole. En ambos casos, nos encontramos con un problema bioético, que involucra la violación de al menos tres de sus principios: beneficencia, no maleficencia y autonomía.

Estimo partir de la siguiente aseveración al respecto, sin temor de que el tema se decante en otro sentido: el individuo intersexual es una persona autónoma, con la única distinción de que su proceso cromosómico y hormonal se completó de forma distinta.

Otro aspecto es el relacionado con el proceso quirúrgico. Las operaciones reconstructivas o de reasignación de sexo son sumamente invasivas e, incluso, en muchos casos pueden resultar traumáticas para el individuo (Alemán Ramírez . 2013)

Lo anterior sin mencionar el hecho de que pueden requerirse otras cirugías posteriores y prácticas o tratamientos adicionales para lograr el objetivo. Por ejemplo, en caso de estenosis postoperatoria se introducen expansores o en el canal vaginal reconstruido, que mide $3 \mathrm{~cm}$ de diámetro y $12 \mathrm{~cm}$ de longitud, el cual se engrapa durante la cirugía y se moviliza cada tres meses, por el plazo de un año, lo que sería necesario para lograr los niveles de elasticidad "naturales" esperados por el médico (Alemán Ramírez ., 2013)

Tal vez el problema más tangible, o el mito más común, lo constituye el aspecto psicológico, que supuestamente afecta tanto a los progenitores como a los individuos intersexuales (Spinola-Castro, 2005). La creencia más generalizada es que los progenitores pueden sufrir muchísimo en caso de procrear un niño o niña intersexual, por la incertidumbre de no saber cómo tratarle hasta su elección de género, lo que incidiría en la formación inicial de la persona menor de edad, en su interacción social con otros menores y la sociedad en general. Esto impulsa a los progenitores a tomar la decisión de someterles a cirugía. Además, priva la opinión -igualmente generalizada- de que, con miras a evitarle a la persona menor de edad problemas de adaptación y aceptación social, lo mejor es tomar las decisiones por ella a temprana edad (Spinola-Castro, 2005).

En muchos de los casos documentados, las personas han manifestado el grave problema que ha resultado, luego de una reasignación temprana de sexo o de género, al modificar registros civiles, nombres, sexo legal, certificados educativos, títulos profesionales, entre otros, lo que ha provocado graves dislocaciones en sus vidas, al descubrir que su género y sexo reasignados no 
corresponden con su identidad cuando adultos. Esto, con el agravante de que el derecho positivo no tiene respuestas oportunas ni leyes dispuestas para reparar el hecho y sus consecuencias, al menos aún no en Costa Rica. Aquí, el cuestionamiento está relacionado con la protección normativa, internacional y local de estas poblaciones, a partir de los derechos humanos, el derecho positivo y los principios bioéticos.

Aún hay otro aspecto importante por considerar: movimientos mundiales que luchan por los derechos humanos de las poblaciones diversas han impulsado iniciativas ante la Organización Mundial de la Salud (OMS) para que la condición de intersexual sea excluida de la lista de enfermedades. Este es un aspecto que debe valorarse en el contexto del tema, pues decidir por una u otra posición podría vulnerabilizar aún más a un grupo de personas intersexuales sin capacidad económica para, una vez definido su género, obtener los tratamientos, pues son altamente onerosos. Por otra parte, no hacerlo podría perpetuar la consideración de la intersexualidad como una "patología que debe tratarse".

\section{Sexo y género: dos conceptos radicalmente distintos}

En un documento denominado Diversidad sexual: conceptos para pensar y trabajar en salud (2011) elaborado como apoyo bibliográfico a las capacitaciones sobre sexualidad, a cargo de Ricardo Duranti, en el marco del proyecto para mejorar la accesibilidad a la prevención, diagnóstico y atención del VIH e ITS de población homosexual, bisexual y trans (coordinado por la Dirección de Sida y Enfermedades de Transmisión Sexual con el apoyo técnico-financiero de ONUSIDA, PNUD, UNFPA y OPS), se utiliza la palabra "sexo" a partir de cuatro grupos: sexo como característica biológica; sexo como comportamiento erótico; sexo como comportamiento social, y sexo como regulador social. Agrega que cuando se alude a sexo:

pensamos solo en su relación con lo genital, pero aún pensando al sexo solamente como biológico, no encontraríamos una entidad única ya que el sexo biológico, que es más que la mera portación de genitales, está constituido por diferentes componentes que tienen, a su vez, características propias: sexo genético (dado por el número de cromosomas); sexo cromatínico (marcado 
por la presencia o ausencia del cuerpo de Barr); sexo hormonal (establecido por el equilibrio entre andrógenos y estrógenos); sexo gonadal (indicado por la presencia de testículos u ovarios); sexo fenotípico (constituido por la morfología del aparato reproductor tanto interno como externo así como por las características morfológicas externas); sexo cerebral (dado por los núcleos del hipotálamo y que son diferentes en los machos y en las hembras, su función es desconocida en el ser humano) (Duranti, 2011, p. 2) (La negrita y el subrayado no son del original).

La definición del sexo, para efectos cualitativos, se da en dos momentos: el nacimiento, cuando se establece un sexo asignado por quienes asisten al momento del parto, guiados por la morfología externa de los genitales, y se denomina sexo de asignación. Esta definición es requerida en el marco legal vigente y se le conocerá como sexo legal a partir de su inscripción. Por otra parte, la acepción de sexo denominada "sexo de crianza" responde a la forma en que los padres (y el entorno social en general) objetivan a los niños y serán los parámetros culturales -masculinos o femeninos- con los que los padres identifiquen a sus hijos, los que lo establecerán

A raíz de estos dos patrones, se construye toda una estructura de normalización social y legal, en la cual aquellos individuos que no encajen son discriminados en todos los entornos sociales, amén de irrespetados en su individualidad y autonomía, así como en su autodefinición de género. Este aspecto tiene mayor relevancia en el caso de los estados intersexuales que en los homosexuales o transexuales, toda vez que la decisión de reasignación de sexo fenotípico es tomada por los progenitores a edades muy tempranas, mientras que homosexuales y transexuales, generalmente, asumen su género en forma autónoma y no por voluntades sustitutas.

De ahí que entre sexo y género podemos hallar una disonancia cognitiva y conceptual, que debe zanjarse. El género es la identidad personal, la cual está determinada no solo por lo cultural, sino por lo social, lo psicológico, lo íntimo, lo impalpable, aquello que quizá en un mundo heteronormativizado resulta inexplicable. El binarismo sexual ha permitido la construcción de un concepto de género de índole biopsicosocial, estableciéndose un parámetro de normalidad donde solamente pueden existir dos sexos: masculino o femenino.

Aquí es importante admitir que en la actualidad este binarismo, como constructo biopsicosocial, ha evolucionado a partir del cuestionamiento sobre 
la veracidad de la afirmación de que solamente existen dos géneros, abriéndose la posibilidad de reconocer que existen más de dos. Todo el sistema binario y heteronormativo de género está representado en los conceptos esbozados por la Organización Mundial de la Salud, por lo que parece que médica y legalmente estamos predestinados, o quizá condenados, a no admitir la existencia de personas que no correspondan con esos criterios normalizadores.

Allí estuvo, muy probablemente, el error del doctor John William Money $^{2}$, en su experimento sobre el sexo aprendido o socialmente construido (caso Reimer), pues él creía que, aunque una persona naciera con sexo fenotípico, hombre o mujer, podría ser reasignado quirúrgicamente, y luego psicológicamente como lo contrario, por medio de la crianza familiar y convivencia social, según el sexo fenotípico reasignado. Como es de suponer, su teoría, con el tiempo, ha resultado incongruente con la realidad y, por lo visto, los conceptos políticamente esbozados por la OMS también, pues el sexo fenotípico y el genotípico pueden no corresponderse, y tampoco construirse desde lo externo como un comportamiento aprendido a partir de lo masculino, lo femenino y sus roles, solamente como una cuestión social.

Es a partir de ello y de esta esfera de intimidad que se llega al punto de inflexión en el tema: ¿Puede el derecho regular estos aspectos de la vida de una persona, limitarlos o restringirlos? ¿Debe estar la persona legalmente sujeta al sistema binario sexual sin perjuicio de su identidad de género? ¿El juramento hipocrático de no hacer daño realmente considera estos aspectos? ¿Estaría exento de responsabilidad un médico respecto a dicho juramento, en caso de no proceder con la intervención en un caso de intersexualidad?

2 John Money (Morrinsville, Nueva Zelanda, 8 de julio de 1921 - Towson, Estados Unidos, 7 de julio de 2006). Psicólogo y médico neozelandés especializado en sexología, emigrado a los Estados Unidos después de la Segunda Guerra Mundial. Su influencia fue determinante en la tesis de la identidad de género y el tratamiento hormono-quirúrgico de la transexualidad. Desde 1951 hasta su muerte, fue profesor de pediatría y psicología médica en la Universidad John Hopkins. Fue el primer integrante honorario de la Asociación Española de Sociedades de Sexología (AEES) y se ha propuesto que el premio de investigación de la Federación Española de Sociedades de Sexología (FESS) lleve su nombre. En 1991 Eli Coleman publicó «John Money, a tribute» en el Journal of Psychology and Human Sexuality, volumen 4, número 2, con la biografía de John Money. En el 2002, recibió la medalla Magnus Hirschfeld de la Sociedad alemana para la investigación científico-social en sexualidad. En el 2009, Money publicó su autobiografía intitulada The thinking traveller, 1946-2003. 


\section{Intersexualidad y principialismo}

El principialismo, en tanto corriente fundante de muchas, sino de todas las disciplinas, resulta vital para analizar los fenómenos sociales, legales, médicos o de cualquier tipo que ocupan la inteligencia humana. Empero, con ello no puede pretenderse, especialmente en bioética, utilizar únicamente este parámetro para evaluar las distintas situaciones que los seres vivos de toda especie pueden enfrentar.

En la realidad multicircunstancial no alcanzaría una sola visión o posición principialista para responder a la compleja situación humana de las personas intersexuales. Por ello, el principialismo de los derechos humanos es otro buen punto de partida, mas no el único enfoque teórico legal que podemos utilizar, en el abordaje de la temática que nos ocupa.

No obstante, el marco teórico que propone el principialismo biomédico y bioético, así como el que plantea el principialismo de los derechos humanos y de los derechos fundamentales convergen en aspectos trascendentales que, tratándose en este tema de uno relativo a la humanidad de los sujetos involucrados, son el punto de partida más apropiado para discutir sobre la intersexualidad.

Algunos aspectos interesantes y no menos curiosos, relativos a ambas teorías principialistas, están relacionados con hechos históricos, por ejemplo: El Estudio Tuskegee, llevado a cabo en la ciudad de Tuskegee, Alabama, investigaba la evolución de la sífilis -sin tratamiento- en esa localidad de los Estados Unidos. Este inició en 1932, con la participación de 600 individuos negros (399 con sífilis y 201 que no contaban con la enfermedad). Su intervención como sujetos investigados fue manipulada con información incorrecta sobre el tratamiento que se les aplicaría, y sin consentimiento informado sobre los beneficios al paciente ni las consecuencias fatales de la enfermedad. Continuó por 40 años, aunque se inició y planificó con una duración de 6 meses, prolongándose hasta 1972, a pesar de la aparición de la penicilina en 1947, como tratamiento admitido para la sífilis; la emisión, en 1947, del Código de Nüremberg, y la Declaración de Helsinki, de la Asociación Médica Mundial de 1964 (Centers for Disease Control and Prevention -CDC-, 2015). 
En 1972 el asunto llegó a la prensa y es hasta entonces que el Parlamento Norteamericano decide crear comisiones nacionales para proteger a quienes participaban en las investigaciones médicas.

En 1974 se creó la National Commission for the Protection of Biomedical and Behavioral Research, con el fin de elaborar principios básicos que regularan la investigación biomédica y el comportamiento con seres humanos, los que culminaron, en 1979, con el Informe Belmont y la creación del Consejo Nacional de Investigación en Humanos (National Human Investigation Board).

Por otra parte, la Segunda Guerra Mundial fue escenario propicio para la idea del Nacional Socialismo, de Adolf Hitler. Este personaje, más allá de establecer un dominio germano de toda Europa por medio de alianzas estratégicas en la parte oriental y occidental, así como en Asia y África, implicaba el surgimiento de una nueva raza aria de características superiores a la existente, por lo que ningún individuo que no calzara en el perfil del nuevo prototipo alemán podría vivir.

Así, a los campos de concentración alemanes en toda Europa fueron enviados judíos, homosexuales, eslavos, discapacitados, gitanos, testigos de Jehová, comunistas, españoles republicanos, sacerdotes católicos y ministros de otras religiones (World Press, 2000-2003).

Durante todo el conflicto, equipos médicos guiados por el doctor Josef Mengele realizaron experimentos con humanos, especialmente, gemelos, personas con heterocromía, personas con enanismo y mujeres embarazadas.

Los experimentos de Mengele con los ojos incluyeron intentos de cambiar el color del iris a través de la inyección de sustancias químicas y el asesinato de personas con heterocromía para extraer sus globos oculares y enviarlos a Berlín para su análisis. A los enanos y a las personas con anomalías físicas les tomaba mediciones corporales, les extraía sangre y dientes sanos, así como les administraba de forma innecesaria drogas y rayos X. Muchas víctimas eran enviadas a la cámara de gas y después sus esqueletos se mandaban a Berlín para continuar con las investigaciones.

Mengele buscó mujeres embarazadas a las que realizaba experimentos antes de enviarlas a la cámara de gas. Esto ocasionó que el final de la guerra fuera seguido por la constitución de la Organización de las Naciones Unidas, el 26 de junio de 1945. 
Posteriormente, en 1948, la Asamblea General de la ONU emitió la Declaración Universal de Derechos Humanos, en la que, en su preámbulo, enuncia una serie de consideraciones respecto a la naturaleza humana, la libertad, la justicia y la protección de la persona humana (ONU Organización de las Naciones Unidas, 2015). Los dos hechos históricos tuvieron, como común denominador, varios aspectos que llaman la atención:

1. Las acciones de cada evento fueron decisiones tomadas por grupos que justificaban, moralmente, su posición y que actuaron al margen del respeto por la humanidad.

2. En ambos casos se produjo la muerte de seres humanos a partir de actos de experimentación con ellos, o del establecimiento de un perfil de normalidad esperada en los sujetos del conglomerado.

3. En ambos casos se sacrificó a poblaciones compuestas por individuos vulnerables.

4. Los intereses velados, tras cada uno de esos hechos, se ubican en la esfera de las posiciones de poder, sea político o profesional (médico).

5. Ambos hechos históricos culminaron con la emisión de importantes documentos conciliatorios y rectores de la conducta humana: la Carta de las Naciones Unidas (1946) y de la Declaración Universal de los Derechos Humanos (1948); el Código de Nüremberg (1947); la Declaración de Helsinki (1964), y el Informe Belmont (1979).

6. En los dos casos, los documentos propugnan la protección de los derechos humanos del individuo y de su bienestar general, en el trato en condiciones de dignidad, libertad y respeto a su autonomía e individualidad.

7. Todos los documentos emitidos son declaraciones de principios morales, de valores de alcance universal y de reconocimiento general, tanto local como global.

De la breve lectura de los documentos citados, se puede concluir que estamos en presencia de declaraciones éticas sobre el tratamiento y consideración que se debe tener a la especie humana por parte de las estructuras de poder hegemónico de cualquier naturaleza. Es aquí donde, curiosamente, el Código de Nüremberg cobra una especial mención, como elemento unificador de ambos planteamientos principialistas, pues, coincidentemente se aplicó como precedente de ulteriores declaraciones e informes que han completado el acervo ético-normativo, tanto de la protección del individuo ante el poder médico y de la investigación biomédica como ante el poder político o militar. 


\section{El principialismo biomédico}

Tom L. Beauchamp y James F. Childress, en la sétima edición de su obra (2013), sostienen que la moralidad particular es aceptable como tal en tanto y cuanto no contradiga o contravenga la moralidad común o universal.

La ética profesional y la moral particular que rige el quehacer de especialistas, en una profesión como la medicina, la abogacía o cualquier otra, puede convertirse en moral particular y será válida, en su aplicación práctica, siempre que no contravenga los términos admitidos por la moral común.

Como tal, la moral particular está sometida a la común, pero, en muchos casos, la moral particular del médico prevalece en la decisión médica de intervención de las personas intersexuales y se impone a una moral común más amplia e integradora de situaciones diversas.

Lo más contradictorio es que el juramento hipocrático y los principios biomédicos desarrollados por Beauchamp y Childress son manipulados por los médicos para normalizar una situación que, desde la moral común, actualmente, resulta aceptada.

No obstante, el principalismo biomédico es medular al establecer un marco referencial para el abordaje de las personas intersexuales.

\section{La teoría de los derechos humanos}

A lo largo del desarrollo temático sobre los derechos humanos, a estos se les ha denominado de distintas formas: -derechos del hombre, derechos naturales del hombre, derechos de la persona humana- y, con miras a su realización, se les ha caracterizado de inmutables, eternos, supratemporales y universales. Bidart Campos (1989) considera que:

a) en la doctrina de los derechos humanos subyace una exigencia ideal; b) la formulación de los derechos humanos se postula como universalmente válida; c) los derechos humanos son superiores y anteriores al Estado y, por eso mismo, inalienables e imprescriptibles, y d) los derechos humanos significan una estimativa axiológica en virtud del valor justicia, que se impone al Estado y al derecho positivo (p. 41). 
De esta forma, en tanto teoría que establece valores supremos, inalienables e imprescriptibles respecto de la humanidad del ser y en tanto estimativa axiológica, esta formulación teórico-práctica se constituye en un primer límite positivo relativo a la garantía de derechos de las poblaciones intersexuales frente a la medicalización de su condición.

\section{La teoría de los derechos fundamentales}

Robert Alexy (2003), en su obra , afirma que "Los derechos fundamentales son en su esencia derechos humanos transformados en derecho constitucional positivo" (p. 26).

Dice Alexy (2003), sobre la teoría de los principios: "De acuerdo con la definición estándar de la teoría de los principios, los principios son normas que ordenan que algo se realice en la mayor medida posible, de acuerdo con las posibilidades fácticas y jurídicas" (p. 95).

Como consecuencia, los principios son mandatos de optimización, que se caracterizan por el hecho de que pueden ser cumplidos en distintos grados y la medida ordenada en que deben cumplirse no solo depende de las posibilidades fácticas, sino también de las jurídicas.

Agrega que la teoría de los principios equivale al principio de proporcionalidad y este implica aquella.

Para Alexy, el deber ser es lo que ha de optimizarse para que, a través de esa optimización, se transforme en un deber ser real. También indica que, en tanto objeto de la optimización, el deber ser tiene que estar en el nivel de los objetos y, por el contrario, los mandatos de optimización o mandatos de optimizar en un metanivel en el cual se establece lo que debe hacerse con lo que está en el nivel de los objetos; sea que el nivel de los objetos (donde se encuentra el deber ser) está sometido al metanivel (mandatos de optimización). Es algo similar al imperativo categórico kantiano.

La teoría de los derechos fundamentales enuncia conductas aceptadas y límites intrínsecos a esas mismas conductas y, en tal relación, la proporcionalidad, la justicia y el respeto surgen como puntos medulares para que una decisión que involucre a un individuo y sus derechos sea tomada con la mayor precaución posible y con criterios de ponderación adecuados. 
De tal suerte, la teoría de los derechos fundamentales, al igual que las otras teorías principialistas citadas, se torna en marco ético normativo para el tratamiento e intervención de las poblaciones intersexuales, con todo lo que el respeto a los mandatos de optimización implica. De lo contrario, serían meras manifestaciones de buena fe sin valor jurídico ni práctico.

\section{Bioética, derecho e intersexualidad}

Los principios biomédicos, luego bioéticos, así como aquellos sobre derechos humanos son el marco referencial para las profesiones relacionadas con el tema de intersexualidad. Por una parte, la medicina, la farmacología, la psicología, la psiquiatría, la endocrinología y la cirugía reconstructiva; por otra, la abogacía y la función legislativa están atravesadas tanto por un orden moral como deontológico y jurídico normativo, que les impone el marco legal y reglamentario de acción a los profesionales del derecho y la medicina. Si bien muchas acciones en el campo médico y en la práctica del derecho son tomadas a partir de criterios morales universalizables, muchas veces la moral particular - de la que nos hablan Beauchamp y Childresssesga la decisión final y descoloca la acción del inspirado por los propios principios teóricos expuestos.

Los principios biomédicos de respeto, no maleficencia y de beneficencia; los bioéticos que extienden los anteriores a la autonomía o independencia y a la justicia; y los de derechos humanos y fundamentales que resguardan la libertad, la justicia, la igualdad, la autonomía de la voluntad, más el derecho a la libre disposición del cuerpo, entre otros, apuntan a que en el caso de la intersexualidad sean cuidadosa y celosamente resguardados.

El problema medular para que esto no suceda es la temprana edad en que se produce la intervención médica de la persona intersexual, y la imperiosa obligación legal que impone el ordenamiento jurídico para definir un "sexo legal" al neonato, con miras a su inscripción.

Otro problema lo representa la situación en sí misma, pues el nacimiento de una persona intersexual -según la literatura consultada y citadaproduce una alteración psicológica en los progenitores ante la presión social del binarismo sexual y la necesaria definición del género. 
Un problema más se produce a partir del juego de las falacias en el argumento normalizador que impone sobre la situación una suerte de "emergencia médica" que "amerita" la intervención inmediata, incurriendo en falacia gradualista al indicar los médicos que "con cada año que pase el problema será mayor"; o bien los argumentos de falso dilema, cuando la pregunta se plantea como "¿es niño o niña?", sin considerar una tercera alternativa o si la "alteración urogenital" es tal que no permite otra "solución" más que la cirugía de reasignación sexual, todo lo cual nos lleva a la falacia de la pendiente resbaladiza. El último de ellos se produce a partir de la inadecuada práctica de la autoridad subrogada y el consentimiento informado.

La situación que envuelve a una persona intersexual no le es exclusiva. Quizás sea una de las condiciones que a más sujetos involucra para la toma de una decisión respecto de la cual el verdadero y único interesado no tiene ni voz ni voto, en razón de la etapa tan temprana en que todo sucede.

Si contamos los individuos que participan en la toma de decisión sobre una cirugía de corazón abierto, cáncer, aborto o participación en una investigación científica, farmacológica o de otra índole, siempre habrá a lo sumo tres sujetos, uno de ellos será el médico, el otro el paciente y, si tuviera pareja, su esposo/esposa, compañero/compañera.

En cambio, en el caso de los intersexuales, al nacer son recibidos por el especialista en ginecología, enfermería obstetra o pediatría, más el padre y la madre. Todos los presentes opinarán con autoridad "suficiente" sobre la situación que recién conocen y la compartirán con los progenitores, quienes, también, pedirán consejo a los médicos sobre qué hacer en ese caso. Mientras tanto, el registrador civil del hospital estará presionando para que se inscriba al individuo recién nacido como hombre o mujer, apelando a la seguridad jurídica y a la protección de los derechos del .

Al calor de esta situación, se pierde de vista por completo que el único interesado y cuya vida a futuro se determinará antes de los 18 meses de edad no puede opinar ni expresar su sentir en relación con su futuro, sexualidad, genitalidad, género y desarrollo psíquico, físico, así como socioafectivo.

Los progenitores pensarán no solo en el bienestar del nuevo integrante de la familia, sino también respecto a su íntima e individual situación. Sus emociones, a partir del consejo médico y las probabilidades futuras de su recién nacido hijo o hija, no les permite centrar la atención en la nueva 
criatura y, apresuradamente, toman la decisión de autorizar cualquier procedimiento necesario para "corregir la anomalía", incluso la "cirugía de reasignación sexual". Es en este momento cuando los principios bioéticos deben ponderarse adecuadamente.

\section{La intersexualidad en Costa Rica}

En el caso costarricense, la intersexualidad no ha dejado de ser considerada una patología o enfermedad por parte de los médicos y los equipos de atención en salud.

Su diagnóstico como enfermedad, en la etapa neonatal y pediátrica, y la preponderancia de criterios fundamentalistas de médicos pediatras reconocidos, como por ejemplo el doctor Orlando Urroz, cirujano pediatra del Hospital de Niños, con 38 años de laborar en esa institución, quien ha sido subdirector y hasta enero de 2015 director médico a. i. de ese hospital y en su vida profesional ha operado - a noviembre de 1997- "a más de 100 niños con esta patología" (Castillo Nieto, 1997), ha decantado el abordaje de estas personas, a edades muy tempranas, a través de procedimientos quirúrgicos que denominan "correctivos y normalizadores".

Digo criterios fundamentalistas refiriéndome a posiciones intransigentes y su aplicación práctica de doctrinas de la misma línea, en este caso no relacionadas con credos o creencias de algún tipo.

Resulta fundamentalista aquella persona que, por temor a enfrentar cambios en lo que hace o debe hacer, prefiere continuar haciéndolo de la misma forma, aunque no sea la mejor o la correcta, pero, es la aceptada, convirtiendo su práctica en una forma de rechazo a las consecuencias seculizadoras de la modernidad.

Y me atrevo a afirmar tanto que el criterio del doctor Urroz es altamente autorizado (ergo poderoso e influyente para el paciente o los progenitores de un neonato intersexual) como que su posición es fundamentalista. Lo afirmo por lo siguiente: su posición de poder ostentada en la estructura político-organizativa del Hospital Nacional de Niños, su exitosa carrera profesional como médico cirujano pediatra, es un importante por considerar para cualquier progenitor que sea atendido por esta autoridad médica nacional. Esto hace que su criterio sea preponderante al momento de abordar 
la atención médica de los menores de edad sometidos a su parecer médico y pesará muchísimo en la decisión de los progenitores sobre si someten o no a cirugía a su recién nacido.

Eso no es negativo, en absoluto. Pero si tal poder lo colocamos en contexto con dos entrevistas periodísticas publicadas en la de y en el periódico, ambos de Costa Rica, y en dos momentos distintos (23 de noviembre de 1997 y 6 de setiembre de 2009, respectivamente, podemos observar claramente una posición fundamentalista.

En el artículo de publicado por Silvia Castillo Nieto, en 1997, el doctor Urroz, como especialista en la atención de personas intersexuales menores de edad, indicó:

los bebés que nacen con estados intersexuales sí tienen sexo, aunque para decir a los padres si el (sic) hijo es varón o mujer es indispensable efectuar una serie de exámenes. El 98 por ciento de los infantes que presentan ambigüedad sexual son niñas y solo el 2 por ciento, hombres. Lo que ocurre es que las chiquitas nacen masculinizadas (pueden tener pene y testículos, o solo un miembro viril pequeño, según la severidad del caso), pero poseen útero y ovarios; además, otros exámenes demuestran que tienen cromosomas femeninos. Actualmente, los expertos recomiendan que la primera cirugía -que consiste en quitar el falo- se haga entre los tres y seis meses de edad. Por lo general, en esa misma intervención el cirujano forma la cavidad vaginal, salvo en casos muy severos en que se necesitan operaciones posteriores. Cuando la afección es a la inversa, los varoncitos nacen con un falo o pene muy pequeño, y usualmente requieren tratamientos con hormonas. Después, la única diferencia entre estos menores y todos los demás es que deberán mantenerse en control médico durante varios años, pero el resto de su vida será normal: llegarán a la pubertad, tendrán relaciones sexuales y las mujeres podrán quedar embarazadas (s. p.).

\section{Igualmente, indica la periodista que:}

este tipo de atención así como las cirugías, solo se realizan en el Hospital Nacional de Niños y están a cargo de un equipo multidisciplinario formado por un endocrinólogo, un genetista, una enfermera, trabajadoras sociales, psicólogas, un anestesiólogo y Urroz Torres como cirujano especializado (s. p.). 
En el artículo publicado en el 2009, en el periódico , a la pregunta del periodista Franklin Arroyo González (2009) sobre qué opina de que algunos digan que lo ideal es permitir que la persona decida su género en la adolescencia, el doctor Urroz responde:

Es una teoría equivocada, nueva, de algunos grupos extremistas. Desde el punto de vista psicológico es inadecuado, para el niño y para la familia. ¿Cómo le va a decir usted a un niño que vaya al kinder y que diga que ni es hombre ni es mujer? Eso no funciona en la sociedad (s. p.).

En ambos artículos periodísticos, aún con 12 años de diferencia entre una y otra entrevista, el mismo médico indica que ante todo caso de intersexualidad la intervención quirúrgica es la norma, con el agravante de que todavía, en el 2009, el doctor Urroz consideraba que permitir que la persona decida su género en la adolescencia es una teoría equivocada, nueva, de algunos grupos extremistas.

Desconoce así que el tema de la intersexualidad y la no intervención quirúrgica de estas personas, en edades tempranas, era un asunto que ya se discutía por Cheryl Chase ${ }^{3}$, desde mediados de 1996, ante la Academia Pediátrica Norteamericana y, en el 2000, por la misma activista, ante el Congreso de la Sociedad Pediátrica Endocrinológica Lawson Wilkins (SPEDLW), prestigioso congreso médico norteamericano.

Igualmente, la Asociación Pediátrica Americana de los Estados Unidos, en su Consensus Statement on Management of Intersex Disorders (Pediatrics, 2006), ya planteaba la posibilidad de no intervenir menores de 18 meses de edad, pues, los primeros signos de la identidad de género surgen a los 3 años y, quizás, se definan hasta la pubertad, por lo que, la cirugía no estaba indicada en todos los casos y, solamente, en aquellos cuya intersexualidad implicara un problema para el desarrollo fisiológico, de salud (riesgos de cáncer o mal funcionamiento genito-urinario) o psicosocial del menor y para su adultez.

De ahí que resulta incomprensible que aún en el siglo XXI, en Costa Rica, el abordaje de estas personas continúe siendo el mismo. ¿No es ello un asunto de fundamentalismo?

3 Activista interesexual norteamericana y fundadora del Intersex Society of North America (ISNA). 
Por otra parte, según información verbal brindada por la doctora Carmen Di Mare Hering, del Área de Bioética del CENDEISS, de la Caja Costarricense de Seguro Social, cada uno de los casos que se presentan son abordados por el Comité de Bioética, el cual -aunque transdisciplinario-no necesariamente está constituido por especialistas en las áreas de abordaje de las personas intersexuales (interdisciplinario) que sí toman decisiones con los progenitores para la intervención quirúrgica antes de los 18 meses de edad.

Además, dejó claro que no existen guías de atención de estas poblaciones en el nivel institucional.

\section{Sobre la incidencia de casos y clasificación estadística}

A pesar de que la incidencia de casos no sea significativa en relación con la población total, desde 1997 al 2014, todos los años se reportó al menos 1 caso y máximo 14 de egresos por "sexo indeterminado" y "seudohermafroditismo" en el Hospital Carlos Sáenz Herrera, Hospital Nacional de Niños, el cual reportó la mayor cantidad de casos de todo el sistema hospitalario (100 del total de 218) (Área de Estadística en Salud, Caja Costarricense de Seguro Social, 2015).

El diagnóstico principal en esos 17 años, en todo el sistema hospitalario nacional, fue de "70 casos de hermafroditismo" no clasificado en otra parte; 1 caso de seudohermafroditismo masculino, no clasificado en otra parte; 3 casos de seudohermafroditismo femenino, no clasificado en otra parte; 2 casos de seudohermafroditismo, no especificado; 138 casos de sexo indeterminado sin otra especificación; y 4 casos de hermafroditismo verdadero 46XX (Área de Estadística en Salud, Caja Costarricense de Seguro Social, 2015)

De 218 casos, 62 fueron hombres; 59 de ellos, menores de 1 año; 1 entre 1 y 4 años; y 2 entre 10 y 14 años (Área de Estadística en Salud, Caja Costarricense de Seguro Social, 2015).

Por su parte, de la misma muestra de incidencia, 52 fueron mujeres; 27 menores de 1 año; 7 entre 1 y 4 años; 5 entre 5 y 9 años; 7 entre 10 y 14 años; 2 entre 15 y 19 años; 3 entre 24 y 44 años; y 1 entre 45 y 64 años (Área de Estadística en Salud, Caja Costarricense de Seguro Social, 2015).

Finalmente, se reportaron como "indefinidos" un total de 104 individuos (Área de Estadística en Salud, Caja Costarricense de Seguro Social, 2015). 


\section{Marco jurídico aplicable}

Según he dicho, la relación directa entre la bioética y los derechos humanos radica, principalmente, en el concepto de dignidad humana, mas no solamente en ella. Principios de igualdad, autonomía, libertad, respeto, independencia, responsabilidad y privacidad, también son componentes esenciales por considerar en el tratamiento de las poblaciones intersexuales. Evidentemente, la integración de la normativa jurídica es fuente importante para la atención de las personas intersexuales y lo es aún más la hermenéutica jurídica de las normas que conforman el Bloque de Constitucionalidad, lo que se debe hacer en aplicación de los principios y los principios bioéticos y una adecuada ponderación de ellos.

\section{Conclusión}

Considerando la media mundial de incidencia de la intersexualidad y que, según las entrevistas sobre el tema, publicadas en y, Costa Rica está entre los primeros países en grado de incidencia de la HSC y de la intersexualidad, resulta incomprensible e incongruente que solamente el $16 \%$ de los diagnosticados con HSC resultaren intersexuales.

Esta cifra está muy por debajo de la media mundial. Si la relación es de 1-2: 2000 individuos nacidos vivos (Wilchins, 2001) y si la población total costarricense es de 4773130 (INEC, 2015), entre 2386 y 1193 habitantes podrán nacer con algún grado de intersexualidad. Sin embargo, esta cantidad de casos se corresponde con el dato de incidencia de HSC (1346), contenido en las estadísticas facilitadas para esta investigación; mas no con la estadística de casos de intersexualidad, la cual asciende a un total de 218, tomando en consideración que la media mundial está relacionada con la intersexualidad y no con la HSC. Sin ánimo de especular sobre este fenómeno, ni sobre si existe o no intencionalidad para alterar u ocultar las estadísticas, podría elaborar un par de situaciones hipotéticas al respecto.

La primera: ocultar o alterar la información para evitar los alcances sociopolíticos de evidenciar una alta incidencia de intersexualidad en el país (como se ha publicitado en 2 artículos periodísticos en 2 momentos distintos) apunta a producir un acto de bien en aplicación del principio de 
beneficencia y no maleficencia, para proteger a estas personas de la estigmatización y la condición de "anormalidad" y de "inadaptación" sociales a la que serían sometidas.

Esta teoría tendría algunas falencias críticas:

a) La ausencia de una correcta cuantificación de casos impide generar políticas públicas en salud, en educación, en información, en inclusividad, para estas poblaciones. Si no existe un significativo número de casos, ¿qué interés tiene revertir lo que se ha venido haciendo (en cuanto a tratamientos y abordaje médico de los casos) o invertir más recursos en atender estas poblaciones?

b) La hipótesis planteada adolece de una ruptura en la parte lógica racional de su estructura, lo que hace dudar de la veracidad de la información que funda la teoría.

La información periodística difundida denuncia que Costa Rica es el segundo país del mundo en "incidencia de intersexualidad"; la media mundial es de 1 o 2 de cada 2000 nacidos vivos y la HSC es la causa más común asociada por las ciencias médicas como causa de la intersexualidad.

Empero, las estadísticas, suministradas por las autoridades de la Caja Costarricense de Seguro Social muestran una correspondencia de los casos de HSC en relación con la media mundial de incidencia de la intersexualidad; pero no en cuanto a los casos de intersexualidad, cuyos datos son realmente bajos. Esto plantea la pregunta lógica racional sobre la consistencia de los datos y cuestiona gravemente la realidad médica y social de esta población, convirtiendo la temática relativa a las personas intersexuales en algo trivial y casi mitológico o surrealista.

Otra hipótesis alcanzada a partir de los hallazgos estadísticos sería:

Los 218 casos presentados en 17 años de estadística, versus los casos reportados de HSC en Costa Rica, reflejan que los casos reportados de HSC no son condicionantes ni determinantes de los presentados en la estadística. Esta hipótesis tendría algunas críticas que la comprometen en su fundamento:

a) Si esta afirmación es cierta, todos los estudios científicos mundiales sobre la intersexualidad y su causa primaria serían falsos. 
b) La media mundial de intersexualidad, en relación con la población total, aplica para todo el resto del mundo menos para Costa Rica, lo que, contrapuesto a la información periodística difundida, resulta contradictorio, pues el país se reporta en los primeros lugares de incidencia de intersexualidad en el mundo.

c) El uso de recursos para el abordaje de la intersexualidad desde la medicina es innecesario y superfluo, ya que lo que se debe atender es la HSC. Esto justificaría la intervención quirúrgica, como primera medida adaptativa del intersexual a la sociedad, y luego se le trataría por problemas de salud relacionados con la HSC.

En cualquier caso, y lejos de continuar cavilando sobre diversas hipótesis, el tema es que, independientemente de que existan 1, 218 o más de 1000 casos de intersexualidad en Costa Rica, el principialismo bioético y de los derechos humanos apunta a que sea la persona intersexual el centro de la atención, no su "enfermedad", como se ha denominado por los médicos y se ha querido sostener y mantener por la medicina nacional durante décadas.

La patologización de la intersexualidad, a partir de discursos y argumentos fundamentalistas y utilitaristas, ha permitido, según se ha podido demostrar con los artículos médicos especializados, publicados en el país, que la cirugía "correctiva” o de reasignación sexual sea la primera ratio para deshacer la "incorrección" natural del individuo respecto del sistema binario de asignación de sexo.

Esta entronización de la patologización pretende buscar la felicidad de una mayoría que se niega a admitir la existencia de personas distintas, y sin importar ni considerar el precio que debe pagar la persona intersexual, especialmente el menor intersexual, al ser sometido a operaciones y tratamientos hormonales que alteran, de una u otra forma, su vitalidad y el sentido de su dignidad como persona integral.

Resulta absolutamente incomprensible la satanización que hacen los médicos de otras alternativas a la cirugía, tachando de extremistas a quienes las impulsan, sin considerar que son los mismos grupos intersexuales interesados los activistas del cambio, y que estos tienen derecho a decidir biomédicamente, bioéticamente y en ejercicio práctico de sus derechos humanos su identidad de género en forma autónoma e independiente. 
Aún resulta más contradictorio el que asociaciones pediátricas internacionales hayan admitido cambiar los procesos médico-quirúrgicos por formas de respeto a la persona, que incluyen primero la observación, dejan la intervención para casos extremos y que, en el entorno local o nacional, aún se siga creyendo y sosteniendo que la forma más adecuada de tratar los estados intersexuales es sometiendo a la persona a cirugía a la menor brevedad posible.

Igualmente, resulta inconcebible que las personas intersexuales no encuentren en la legislación nacional las garantías de protección de su personalidad jurídica, sus derechos autónomos de decisión, su libertad de definir su género y sexo, su derecho a ser respetados integralmente y a ser tratados con dignidad, sin estigmas ni discriminaciones. Todo ello, a pesar de que Costa Rica ocupa el número 24 en el mundo en el índice de respeto por los derechos humanos.

El ejercicio médico impone altos niveles éticos que, a su vez, implican la observancia y respeto de los principios bioéticos estudiados. Igualmente, el ejercicio de los derechos humanos, en tanto ética aplicada, también exige la observancia de principios éticos claramente establecidos y aquí estudiados.

De las manifestaciones de la Dra. Carmen Di Mare Hering, del Área de Bioética del CENDEISS, de la Caja Costarricense de Seguro Social, queda demostrada la inexistencia de protocolos y guías de atención de las personas intersexuales, desde su nacimiento hasta su muerte, lo que es una grave falencia del sistema de seguridad social y de salud pública, pues, se cohonesta el ilegítimo e inmoral abordaje patológico de estas personas, cosificándoles y convirtiéndoles en sujetos de tortura y objetos de experimentación, todo revestido de un halo de legitimidad por un paternalismo médico mal aplicado y mal entendido.

La Caja Costarricense de Seguro Social y el Ministerio de Salud como ente rector en salud deberían iniciar una campaña informativa sobre la incidencia de la HSC y su efecto en el , con miras a informar a la población sobre la existencia de la intersexualidad en la sociedad costarricense y así visibilizarla. Esto erradicaría el morbo y los estigmas que pesan sobre esa población y facilitaría su inserción, inclusión social y reconocimiento político en una sociedad que se niega a aceptar esta realidad. Ambas instituciones deberían iniciar reuniones y estudios para la elaboración de un catálogo de estados intersexuales. 
Saber la media costarricense respecto de la mundial, la relación que existe entre la HSC y la intersexualidad en Costa Rica, así como los tipos de intersexualidad específica que se presentan en los recién nacidos costarricenses permitirá discriminar, en el nivel de protocolos y guías médicas, cuáles casos podrían ameritar una intervención quirúrgica y cuáles no.

La Caja Costarricense de Seguro Social, asociada con el Ministerio de Salud, debería establecer políticas públicas claras de atención de esta población y sus familiares, tanto al implementar las guías nacionales para atender a estas personas por los equipos médicos, como por los profesionales en trabajo social, psicología, endocrinología, pediatría, entre otros, relacionados con su abordaje en el sistema de salud y de seguridad social nacional a lo largo de sus vidas.

El Ministerio de Educación, en conjunto con el Ministerio de Salud, deberían establecer políticas educativas en torno a la intersexualidad, que permitan informarse sobre esta realidad. Debe establecerse que su abordaje sea con total naturalidad, que es exactamente como sucede en el proceso de formación del feto, para que no se estigmatice ni satanice, impidiendo de esa forma su patologización.

Igualmente, debe separarse, a partir de guías educativas, el caso de la homosexualidad, la transexualidad y la bisexualidad del intersexualismo, estableciendo las diferencias básicas entre esta y aquellos estados, como por ejemplo, que la condición de intersexualidad es una condición genético-biológica heredada de los progenitores, sobrevenida por preexistencia de determinantes en la madre.

La apertura sociológica y psicológica en la sociedad costarricense, a través de la reeducación en el tema de la intersexualidad, permitirá la concientización sobre la existencia de individuos distintos a la "normalidad" o norma establecida desde el binarismo sexual, lo que instará el cambio paradigmático en relación con los intersexuales. Esto les facilitará ser aceptados e incluidos socialmente sin injerencia de ese patrón heteronormativo, abstrayendo, a la vez, a los progenitores de la toma de decisiones respecto de menores intersexuales, generando espacios de respeto hacia ellos y a sus determinaciones.

El Colegio de Médicos y Cirujanos de Costa Rica, asociado con autoridades de la Caja Costarricense de Seguro Social, el Ministerio de Salud, el Ministerio de Educación y otros colegios profesionales como el de Psicólogos, 
Sociólogos, Abogados, Psiquiatras, Farmacéuticos, debe emprender una campaña de concientización sobre el uso de conceptos y discursos patologizantes, modificar los argumentos a partir de usos lingüísticos de vocablos más centrados en la persona al igual que en su dignidad humana y no en la enfermedad, con el fin de evitar encasillamientos, estigmas y discriminaciones en relación con las personas intersexuales.

Mucho se ha discutido internacionalmente sobre este tema y, por ello, creo que el diálogo nacional debe abrirse no solo para establecer un uso común y estándar internacional, sino para mostrar la capacidad de un país y sus profesionales en proponer, quizá, un nuevo concepto que evite la patologización de las personas intersexuales.

\section{Bibliografía}

Alemán Ramírez, R., Céspedes Durán, L., Fernández Vaglio, R., Herrera Rodríguez, A., Sánchez Villalobos, N., Solar Del Valle, T. y Soto Zúñiga, M. (2013). Desórdenes del Desarrollo Sexual y Cirugía Correctiva. Recuperado el 20 de 01 de 2018 de http://www.scielo. sa.cr/scielo.php?script=sci_arttext\&pid=S1409-00152013000200008\&lng=en\&tlng=es. Alexy, R. (2003). Colombia: Universidad Externado de Colombia.

Área de Estadística en Salud, Caja Costarricense de Seguro Social. (2015). San José, Costa Rica.

Arroyo González, F. (6 de setiembre de 2009). De cinco a diez bebés nacen como ella aquí. . Beauchamp, T. y Childress, J. (2013). New York: Oxford University Press.

Berger, L. y Luckmann, T. (2001). Argentina: Amorrortu.

Bidart Campos, G. J. (1989). México: Instituto de Investigaciones Jurídicas.

Bordes Solanas, M. (2011). Madrid: Ediciones Cátedra (Grupo Anaya S. A.).

Castillo Nieto, S. (23 de noviembre de 1997). Entonces, ¿es varón o mujer? .

Centers for Disease Control and Prevention CDC. (04 de 2015). Obtenido de http://www. cdc.gov/tuskegee/timeline.htm

Duranti, R. (2011). Argentina: ONUSIDA.

González Vicen, F. (1969). Madrid: Anuario de Filosofía del Derecho.

ISNA. (31 de 10 de 2014). Intersex Society of North America. Recuperado de http://www. isna.org/faq/concealment

INEC. (10 de abril de 2015). Instituto Nacional de Estadística y Censos. Recuperado de http://www.inec.go.cr/Web/Home/GeneradorPagina.aspx

Katz, R., Russell, S., Kegeles, S. y Kressin, N. (Noviembre de 2006). The Tuskegee Legacy Project: Willingness of Minorities to particpate in Biomedical Research, 17 (4), 698-715.

MEDLINEPLUS. (12 de agosto de 2014). MedlinePlus. Recuperado de http://www.nlm. nih.gov/medlineplus/spanish/ency/article/001669.htm 
OMS. (2002). Organización Mundial de la Salud Recuperado de http://www.who.int/ gender/mainstreaming/ESPwhole.pdf

ONU. (4 de abril de 2015). Naciones Unidas. Recuperado de http://www.un.org/es/documents/charter/preamble.shtml

Pacheco de Arauz, G., Piza Escalante, J., Arauz Pacheco, C. y Fallas F., P. (1984). Frecuencia de las malformaciones congénitas en las autopsias del Hospital Nacional de Niños. San José.

Spinola-Castro, A. M. (2005). A importancia dos Aspectos Éticos e Psicológicos na Abordagem do Intersexo. 49(1). Recuperado de https://dx.doi.org/10.1590/ S0004-27302005000100007

Wilchins, R. (2001). A girl's right to choose: intersex childern and parents challenge narrow standards on gender. 34(2)

World Press. (2000-2003). Historia de la Segunda Guerra Mundial Recuperado de https:// futurodelmundo.files.wordpress.com/2009/01/historia-de-la-segunda-guerra.pdf 